\title{
Emergence of communities in weighted networks
}

\author{
Jussi M. Kumpula ${ }^{1}$. ${ }^{\text {J Jukka-Pekka Onnela }}{ }^{2,3,1}$, Jari Saramäki ${ }^{1}$, Kimmo Kaski ${ }^{1}$, and János Kertész ${ }^{1,4}$ \\ ${ }^{1}$ Laboratory of Computational Engineering, Helsinki University of Technology, P.O. Box 9203, FIN-02015 HUT, Finland \\ ${ }^{2}$ Physics Department, Clarendon Laboratory, Oxford University, Oxford OX1 3PU, U.K. \\ ${ }^{3}$ Saïd Business School, Oxford University, Oxford OX1 1HP, U.K. \\ ${ }^{4}$ Department of Theoretical Physics, Budapest University of Technology and Economics, Budapest, Hungary
}

(Dated: October 22, 2018)

\begin{abstract}
Topology and weights are closely related in weighted complex networks and this is reflected in their modular structure. We present a simple network model where the weights are generated dynamically and they shape the developing topology. By tuning a model parameter governing the importance of weights, the resulting networks undergo a gradual structural transition from a module free topology to one with communities. The model also reproduces many features of large social networks, including the "weak links" property.
\end{abstract}

PACS numbers: 89.75.Hc, 87.16.Ac, 89.65.-s,89.75.Fb,89.75.-k

Network theory has undergone a remarkable development over the last decade and has contributed significantly to our understanding of complex systems, ranging from genetic transcriptions to the Internet and human societies [1, 2]. Many complex networks are structured in terms of modules, or communities, which are groups of nodes characterized by having more internal than external connections between them. Such a mesoscopic network structure is expected to play a concrete functional role. Consequently, it is an important problem to understand how the communities emerge during the growth of the network. Apart from these issues of topological nature, it is important to realize that many complex networks are weighted, i.e., the interaction between two nodes is characterized not only by the existence of a link but a link with a varying weight assigned to it. There are a number of examples, like traffic, metabolic or correlation based networks, which provide ample evidence that the weights have to be included in their analysis. In many cases the weights affect significantly the properties or function of these networks, e.g., disease spreading [3], synchronisation dynamics of oscillators [4], and motif statistics [5]. It is natural to expect that weights have an influence on the formation of communities, which is the very issue of our study.

Earlier, coupled weight-topology dynamics have been used successfully in transport networks modeling [6], which, however, does not lead to community structure. We show that there are mechanisms, by which weights play a crucial role in community formation. While we believe this to be quite a general paradigm for community formation, we have chosen to explore it within the realm of social systems where large datasets have enabled looking into both the coupling of network topology and interaction strengths and properties of communities [7, 8, 9]. Understanding how the underlying microscopic mechanisms translate into mesoscopic communities and macroscopic social systems is a key problem in its own right and one that is accessible within the scope of sta- a)

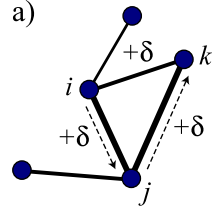

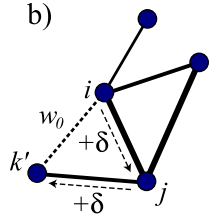

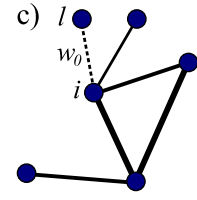

FIG. 1: The model algorithm. (a): a weighted local search starts from $i$ and proceeds first to $j$ and then to $k$, which is a neighbor of $i$. (b): the local search from $i$ ends to $k^{\prime}$, which is not a neighbor of $i$. In this case link $w_{i k^{\prime}}$ is established with probability $p_{\Delta}$. (c): node $i$ creates a random link to random node $l$ with probability $p_{r}$. In cases a) and b) the weights of involved links are increased by $\delta$.

tistical physics.

Large scale social networks are known to satisfy the weak links hypothesis [10] with the implication that weak links keep the network connected whereas strong links are mostly associated with communities [24]. This weighttopology coupling results from the microscopic mechanisms that govern the evolution of social networks. Network sociology identifies (a) cyclic closure and (b) focal closure as the two fundamental mechanisms of tie formation [11. Cyclic closure refers to forming ties with one's network neighbors - "friends of friends". Experimental evidence indicates that the probability of cyclic closure decreases roughly exponentially as a function of the geodesic distance [12. Focal closure, in contrast, refers to forming ties independently of the geodesic distance and is attributed to forming social ties through shared activities (hobbies etc.) [11. These two mechanisms form the basis of the topological rules of our model. As for the weights, we have chosen a simple scenario in which new ties are created preferably through strong ties, every interaction making them even stronger.

We consider a fixed size network of $N$ nodes, where links are created in two ways: First, in time interval $\Delta t$ each node having at least one neighbor starts a weighted local search for new acquaintances, Fig. 1(a,b). More 


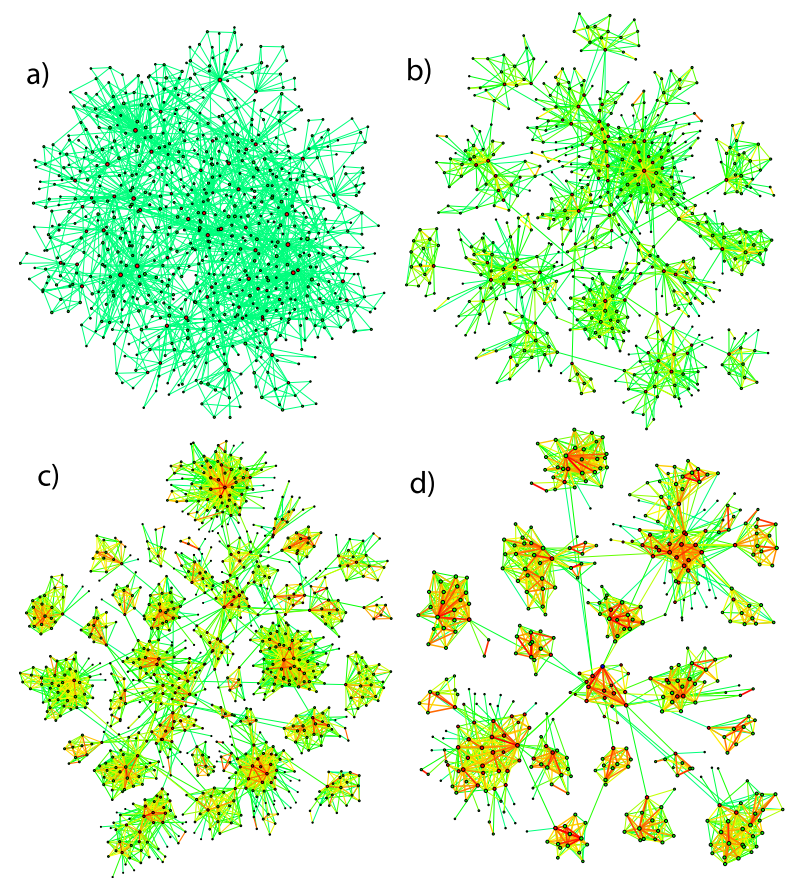

FIG. 2: (Color online) Snowball samples 13 of networks with (a) $\delta=0$, (b) $\delta=0.1$, (c) $\delta=0.5$, and (d) $\delta=1$. Link colors change from green (weak links) to yellow and red (strong links).

specifically, node $i$ chooses one of its neighbors, node $j$, with probability $w_{i j} / s_{i}$, where $w_{i j}$ is the weight of the link connecting $i$ and $j$ and $s_{i}=\sum_{j} w_{i j}$ the strength of $i$. If the chosen node $j$ has other neighbors apart from $i$, it chooses one of them, say $k$, with probability $w_{j k} /\left(s_{j}-w_{i j}\right)$. Therefore, the search favors strong links. If there is no link between $i$ and $k$, it is established with probability $p_{\Delta} \Delta t$ such that $w_{i k}=w_{0}$. If the link exists, its weight is increased by an amount $\delta$. In both cases, $w_{i j}$ and $w_{j k}$ are also increased by $\delta$. We call this attachment mechanism local attachment (LA), and it corresponds to a special case, triadic closure, of the above mentioned cyclic closure mechanism. Second, if a node has no links, or otherwise with probability $p_{r} \Delta t$, it creates a link of weight $w_{0}$ to a random node, Fig. 1(c). This mechanism corresponds to establishing a new interaction outside the immediate neighborhood of the chosen node, analoguously to focal closure, and we call it global attachment (GA). Finally, any node can be removed with probability $p_{d} \Delta t$ by the node deletion (ND) mechanism, in which case also the adjacent links are removed. The removed node gets replaced by a new node, such that the size of the system remains fixed at $N$. ND is the only mechanism that decreases the number of links in the model and leads to exponentially distributed lifetime $\tau$ for nodes and $\tau_{w}$ for links, averages of which are

$$
\begin{aligned}
\langle\tau\rangle & =\left(p_{d} \Delta t\right)^{-1} \\
\left\langle\tau_{w}\right\rangle & =\left(2 p_{d} \Delta t\right)^{-1} .
\end{aligned}
$$

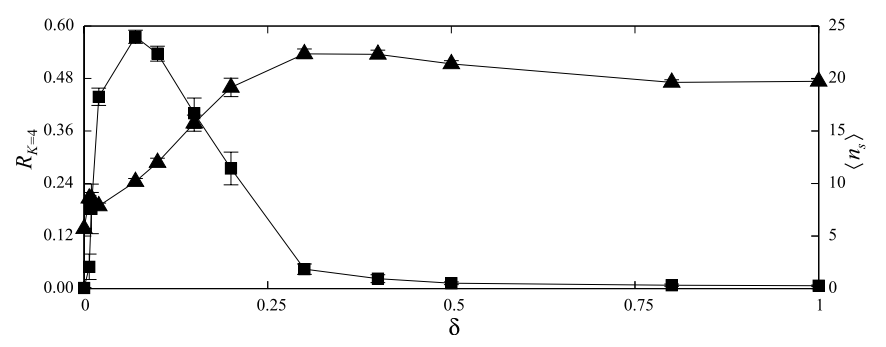

FIG. 3: $R_{k=4}(\square)$ and $\left\langle n_{s}\right\rangle(\triangle)$ as a function of $\delta$. Results are averaged over 10 realizations of $N=5 \times 10^{4}$ networks. Error bars are measured standard deviations.

The model was studied by simulations, which were started from a seed network of $N$ nodes without any links. Subsequent changes due to LA and GA mechanisms were updated using parallel update, followed by the ND step. We set $\Delta t=1$ and $w_{0}=1$ without loss of generality. The time scale is fixed by the death rate $p_{d}$, which was set to $10^{-3}$ corresponding to $\langle\tau\rangle=10^{3}$ time units. The random link probability was set to $p_{r}=5 \times 10^{-4}$, corresponding to adding on average one random link for each node during average node lifetime. The network algorithm was found to reach steady state in $\sim 10-20$ average node lifetimes, after which all measured characteristics were found to be stationary. The following results were obtained by running the simulations for $25 \times 10^{3}$ time steps, i.e., 25 average node lifetimes.

The weights enter the model dynamics through parameter $\delta$. In order to compare networks resulting from different values of $\delta$, we have chosen to keep the average degree $\langle k\rangle \approx 10$ constant. Thus, the number of links is roughly equal in all networks and changes in the structure result solely from restructuring of the links. Keeping $\langle k\rangle$ constant requires adjusting $p_{\Delta}$ for each $\delta$, which can be done easily as their dependence is a smooth monotonous function [25]. When $\delta=0$ we obtain unweighted networks that resemble those obtained by certain older models [14, 15] without apparent community structure. However, increasing $\delta$ results in denser networks in which communities clearly appear as seen in Fig. 2. This can be attributed to the effect of $\delta$ on the LA mechanism. The higher the value of $\delta$, the more trapped the local searches become, i.e., they repeatedly follow the same links, simultaneously increasing the weights of these links and the associated triangles, which in turn further enhances the trapping effect. Thus, in the transient phase of the model dynamics prior to the stationary state, any emerging triangle starts to rapidly accumulate weight, acting as a nucleus for community formation.

We consider first the topological structure of the model networks and study the communities using the $k$-clique percolation method [8, 16]. This avoids the problems of modularity-based methods [17, 18, 19, which may not properly resolve communities if their size distribution is broad. The LA mechanism, which is mainly responsible 
for introducing new links, generates at least one triangle per added link. Therefore, we focus on 4-cliques, the smallest non-trivial cliques beyond triangles. Figure 3 shows the relative largest community size $R_{k=4}$ and average community size excluding largest community $\left\langle n_{s}\right\rangle$ for $\delta \in[0,1]$. When $\delta=0$ communities are mainly very small, $\left\langle n_{s}\right\rangle \sim 6$ and the largest ones contain $\sim 50$ nodes. Increasing $\delta$ changes the network structure significantly. At first, the network becomes homogeneous in the sense that the 4-cliques percolate through most of the system, but as $\delta$ becomes larger the nodes begin to condensate in tighter communities. This can be seen as the increase in $\left\langle n_{s}\right\rangle$ and simultaneous decrease in $R_{k=4}$. When $\delta \gtrsim 0.2$ the network contains communities whose $\left\langle n_{s}\right\rangle \sim 20$ while the largest ones consist of several hundred nodes. The probability for communities of size larger than 50 to occur is several orders of magnitude higher for $\delta \gtrsim 0.2$ than for $\delta=0$. Similar results were obtained for $k$-clique percolation with $k=3$ and $k=5$. The community size distribution was found to be stable after $\sim 10$ average node lifetimes. This can be understood in the large $\delta$ limit by considering the change in the size $N_{s}$ of community $s$. When $\delta$ is large the LA process mostly follows the strong within-community links and we can assume that community merging is rare. We can now estimate the change in $N_{s}$ as

$$
\frac{\mathrm{d} N_{s}}{\mathrm{~d} t}=-p_{d} N_{s}+p_{d} N \frac{N_{s}}{N}=0,
$$

where the first term on the r.h.s. follows from the fact that each of the $N_{S}$ nodes in $s$ is deleted with probability $p_{d}$, and the second reflects the fact that of the all $p_{d} N$ deleted nodes, a fraction of $N_{s} / N$ will form an initial, random link to community $s$, thus becoming a member of that community at subsequent time steps by the LA process. This shows that once the algorithm has reached a state in which most local searches remaining in the initial community is valid, the community size distribution remains constant. Figures 2 and 3 indicate that $\delta \gtrsim 0.5$ can already be regarded as "large", because the communities are tight and increasing $\delta$ does not change them significantly.

Next, we consider the effect of $\delta$ on weight-topology correlations and study the network structure with link percolation. Weak links hypothesis implies that links within communities are strong whereas links between them are weak. Therefore, if the network has such a structure, it should disintegrate faster when links are removed in ascending than in descending order of weight, as observed in [7. Here we remove links from the network in both orders while monitoring the network properties as a function of fraction of removed links $f$, which acts as a control parameter. We have measured the relative size of the giant component $R_{L C C}$ serving as an order parameter, the 'normalized susceptibility' $\tilde{s}=\sum n_{s} s^{2} / N$, where $n_{s}$ is the number of components of size $s$ and the

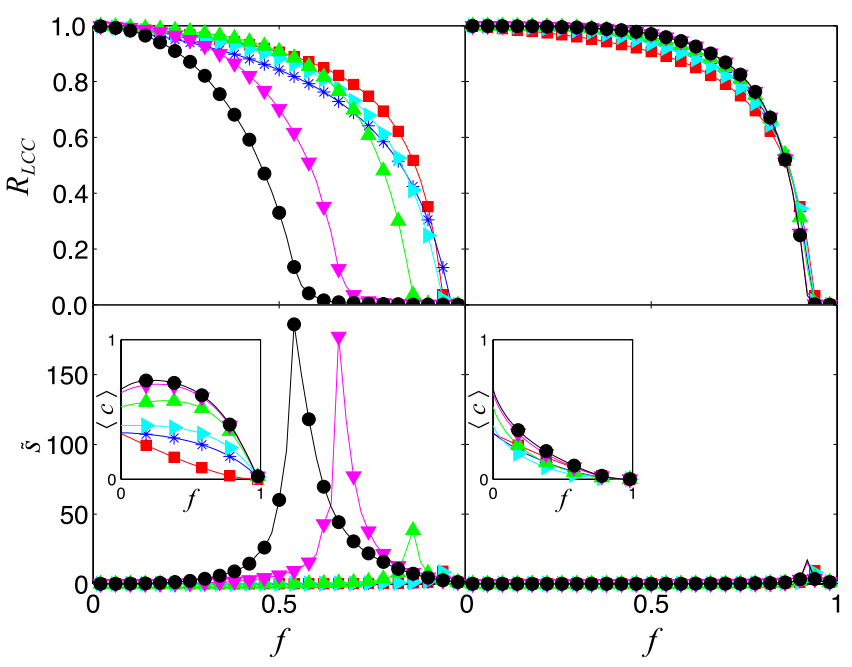

FIG. 4: (Color online) $R_{L C C}$ and $\tilde{s}$ for link percolation. Left: weak links removed first, right: strong links removed first. Insets: Average clustering. Results are averaged over 10 realizations of $N=5 \times 10^{4}$ networks. Values of $\delta$ are $0(\square)$, $1 \times 10^{-3}(*), 1 \times 10^{-2}(\triangleright), 0.1(\triangle), 0.5(\nabla)$, and $1(\circ)$.

sum is taken over all but the largest component, and the average clustering coefficient $\langle c\rangle[20$. Figure 4 shows link percolation results for networks for $\delta \in[0,1]$. For small values of $\delta$ it appears that there is no community structure compatible with the weak links hypothesis, as the giant component is destroyed at the same point for both removal orders and $\tilde{s}$ remains very small. However, when $\delta \gtrsim 0.1$ the networks start to break faster when weak links are removed first and $\tilde{s}$ developes a finite signature of divergence, indicating that the weak links serve as bridges connecting communities. This is also corroborated by the the rapid decrease in $\langle c\rangle$ when strong links are removed first (see inset). This effect was also verified similarly to Refs. [7, 21] using the topological overlap measure, which was found to increase clearly as a function of link weight when $\delta \gtrsim 0.1$ (not shown).

Finally, as our model is inspired by mechanisms of social network formation, we investigate whether it reproduces also other properties of social networks. The picture emerging from analysis of a number of large data bases [7, 21, 22, 23] shows several common features: $i$ ) degree distributions are skewed, ii) high-degree nodes are often connected to other high-degree nodes (assortative mixing), $i i i)$ the average clustering coefficient $\langle c\rangle$ is high compared to random networks, and $i v$ ) the average shortest path lengths are small (the small-world property). Figure 5 presents the basic distributions for network quantities with $\delta \in[0,1]$. The degree distribution was found to be almost independent of $\delta$. It has a quite fast decaying tail with the maximum degree $\sim 10^{2}$ for networks with $\langle k\rangle \approx 10$ and can be fitted reasonably with an exponential distribution. The networks show high clustering behaving almost as $c(k) \sim 1 / k$. The 

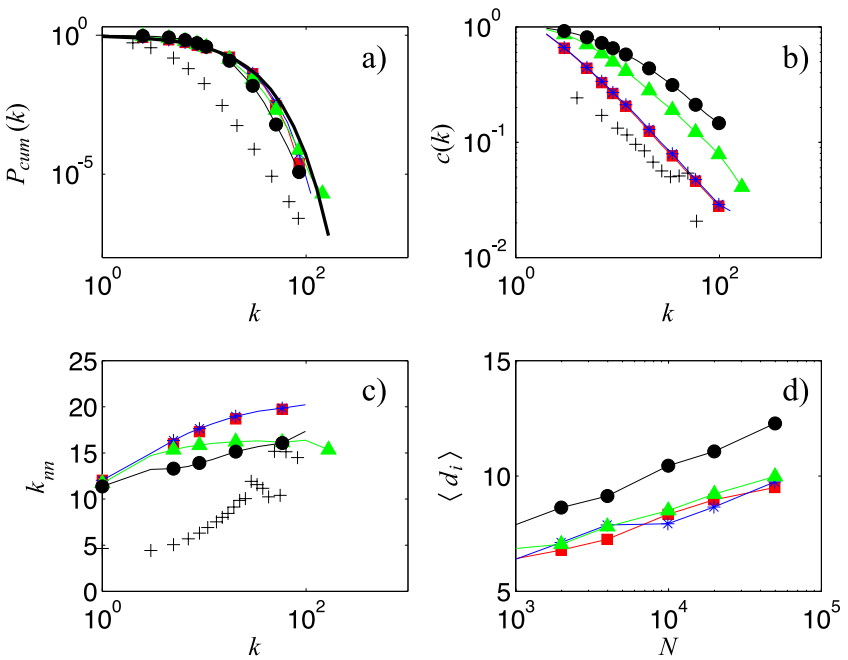

FIG. 5: (Color online) The model meets criteria $(i)-(i v)$ for social networks (see text) when $\delta \in[0,1]$. (a) Degree distribution, (b) clustering, (c) average nearest neighbor degree $k_{n n}$, and (d) sampled network diameter as a function of network size $N$. Results for (a), (b), and (c) are averaged over 10 realizations of $N=5 \times 10^{4}$ networks. The corresponding distributions for the mobile phone call network studied in Ref. 7] are marked by + (note that this network has average degree $\langle k\rangle=3.14$ ), the exponential fit in (a) is shown as a solid line, other symbols are as in Fig. 4

model networks are also found to be assortative and the diameter grows as $\log N$, Fig. 5 (c,d).

In this paper we have introduced a simple weighted network model in which the weights are an essential part of the microscopic mechanisms. They establish a coupling between network structure and interaction strengths: addition of a new link depends on the existing weights, and once a new link is added the weights that led to its formation are strengthened. Communities will emerge only if this strenghtening is large enough, i.e., if nodes favor sufficiently their strong connections in the process of establishing new ones. Our study support the notion that communities result from initial structural fluctuations, which become amplified by repeated application of the microscopic processes. In addition to fulfilling the standard topological properties of social networks, the model networks exhibit a coupling between network topology and interaction strengths, which is compatible with the weak links hypothesis. Most importantly, this model provides a starting point for understanding the formation of communities in weighted networks.

Acknowledgements: J.M.K., J.-P.O., J.S. and K.K. acknowledge the Academy of Finland, the Finnish Center of Excellence program 2006-2011, proj. 213470. J.-P.O. is supported by a Wolfson College, University of Oxford,
Junior Research Fellowship. J.K. was partially supported by OTKA T049238 grant.

* Electronic address: jkumpula@lce.hut.fi

[1] M. E. J. Newman, A.-L. Barabási, and D. J. Watts, The Structure and Dynamics of Networks (Princeton University Press, 2006).

[2] G. Caldarelli, Scale-Free Networks: Complex Webs in Nature and Technology (Oxford University Press, 2007).

[3] V. Colizza, A. Barrat, M. Barthélemy, and A. Vespignani, Proc. Natl. Acad. Sci. U.S.A. 103, 2015 (2006).

[4] M. di Bernardo, F. Garofalo, and F. Sorrentino, condmat/0504335 (2005).

[5] J.-P. Onnela, J. Saramäki, J. Kertész, and K. Kaski, Phys. Rev. E 71, 065103(R) (2005).

[6] A. Barrat, M. Barthélemy, and A. Vespignani, Phys. Rev. Lett. 92, 228701 (2004).

[7] J. P. Onnela, J. Saramäki, J. Hyvönen, G. Szabó, D. Lazer, K. Kaski, J. Kertész, and A.-L. Barabási, Proc. Natl. Acad. Sci. U.S.A. 104, 7332 (2007).

[8] G. Palla, I. Derényi, I. Farkas, and T. Vicsek, Nature 435, 814 (2005).

[9] G. Palla, A.-L. Barabási, and T. Vicsek, Nature 446, 664 (2007).

[10] M. Granovetter, Am. J. Soc. 78, 1360 (1973).

[11] G. Kossinets and D. J. Watts, Science 311, 88 (2006).

[12] D. J. Watts, private communication (2007).

[13] S. H. Lee, P.-J. Kim, and H. Jeong, Phys. Rev. E 73, 016102 (2006).

[14] M. Marsili, F. Vega-Redondo, and F. Slanina, Proc. Natl. Acad. Sci. U.S.A. 101, 1439 (2004).

[15] J. Davidsen, H. Ebel, and S. Bornholdt, Phys. Rev. Lett. 88, 128701 (2002).

[16] I. Derényi, G. Palla, and T. Vicsek, Phys. Rev. Lett. 94, 160202 (2005).

[17] M. E. J. Newman and M. Girvan, Phys. Rev. E. 69, 026113 (2004).

[18] S. Fortunato and M. Barthélemy, Proc. Natl. Acad. Sci. U.S.A. 104, 36 (2007).

[19] J. M. Kumpula, J. Saramäki, K. Kaski, and J. Kertész, Eur. Phys. J. B 56, 41 (2007).

[20] D. J. Watts and S. H. Strogatz, Nature 393, 440 (1998).

[21] J. P. Onnela, J. Saramäki, J. Hyvönen, G. Szabó, M. de Menezes, K. Kaski, A.-L. Barabási, and J. Kertész, New J. Phys. 9, 179 (2007).

[22] M. C. González, H. J. Herrmann, J. Kertész, and T. Vicsek, Physica A 379, 307 (2007).

[23] M. E. J. Newman, Proc. Natl. Acad. Sci. U.S.A. 98, 404 (2001).

[24] The weak link hypothesis [10] was recently proved on societal-level one-to-one communication networks [7, 21]

[25] Keeping $p_{d}$ and $p_{r}$ constant, the balance of link addition and deletion depends on the rate at which new links are formed in the LA process. Evidently this depends on the LA probability, $p_{\Delta}$, but also on the probability to end up at a node not connected to the starting node. Increasing $\delta$ constrains the searches to 'familiar paths', decreasing this probability, and consequently leads to a lower degree. 